\title{
NANOBOTS: APPLICATIONS IN BIOMEDICAL
}

\author{
Shailesh \\ M. Tech, Scholar, Embedded System \\ Lingayas Vidyapeeth, \\ Faridabad, India
}

\begin{abstract}
The utilization of nanotechnology with the new electronic materials prompted the advancement of nanorobots. Nanorobots in the nano scale locale are equipped for entering the phone for conclusion, treatment and medical procedure. Because of its wide reach of uses, nanorobots are planned with explicit materials furthermore, plan innovations. Since the biocompatible materials are utilized in the plan of nanorobots, they are synthetically dormant and the issue of poisonousness is stayed away from. The nano scale size permits the designated conveyance of medications to the particular location without influenced the ordinary encompassing cells. Nanorobots have the ability to move uninhibitedly in the circulation system because of the Brownian movement. This paper plainly clarifies the different biomedical utilizations of the nanorobots
\end{abstract}

Keywords-Nanorobots, identifications, medication, surgical procedure

\section{INTRODUCTION}

Nanorobotics is an arising field of nanotechnology which manages plan and development of gadgets at an nuclear, atomic or cell level. These theoretical nanorobots will be minuscule and would cross over inside the human blood. As these nanorobots would have exceptional sensors to distinguish the objective atoms, it very well may be modified to finding and treat different indispensable sicknesses [1]. Nanorobots would comprise any "shrewd" arrangement able to do incitation, detecting, flagging, data handling, insight, control and multitude conduct at nano scale $\left(10^{-9} \mathrm{~m}\right)$. Nanomedicine opens up the prospect of amazing new technologies for treating human illnesses and improving human biological structures. Nanomedicine is the use of sub-atomic devices and sub-atomic information on the human body to diagnose, cure, and prevent illness and terrible harm, to relieve pain, and to save and improve human health. [2]. A nanorobot is a minuscule designed to carry out a specific task with nanoscale precision. Nanorobots or nanoids are the alternative names for them. The nanorobot's design is based on organic microbe models. Carbon will most likely be the most common component used in nanorobot creation, and it will most likely be housed as a jewel/ diamondoid which includes pure precious stone and translucent allotrope of carbon) or

\author{
Kapil Kumar \\ Assistant Professor, Electronics and Communication \\ Lingayas Vidyapeeth \\ Faridabad, India
}

fullerene nanocomposites. A nanorobot can be made of mechanical parts like bearing, gears, engines and so on The external shell of nanorobot is probably going to be developed utilizing diamondoid material because of their latent properties, high warm conductivity and strength. The super-smooth surfaces could lessen the odds of setting off the body's resistant framework. The nanoscale gears and different parts intended for particular purposes could be built utilizing components like hydrogen, sulphur, oxygen, nitrogen, silicon and so on [3]. The ability to build nanorobots may be aided by the newest innovations and techniques in manufacturing, computation, transducers, and control. High precision and a business method for constructing early nanodevices and circuits are provided by CMOS VLSI configurations using profound brilliant lithography gives high accuracy and a business route for assembling early nanodevices and nanoelectronics frameworks [4]. To approve plans and to accomplish an effective execution, the utilization of the coordinated circuit (IC) manufacturing business, VHDL (Verification Hardware Description Language) has been the most generally known technique [5]. With nanorobots imparted aggregately for greater distances using sound sensors, CMOS with submicron $\mathrm{SoC}$ architecture may be used for astonishingly low force usage [6]. The many utilizations of nanorobots in the fields of medical procedure, nervous system science, dentistry, haematology, microbiology, malignancy therapy, and quality treatment are the focus of this review.

\section{BIOMEDICAL UTILIZATIONS OF NANOROBOTS}

\section{A. Surgery using Nanorobots}

In the conventional medical procedure strategies, the specialists will cut the solid tissues and uncover the organs or parts to be worked. Because of the advances in the clinical innovation, insignificantly intrusive careful techniques are being utilized broadly. The benefit of negligibly obtrusive medical procedure incorporates less scarring, less agony, decreased contamination rates and quicker restoration. With the scaled down size, nanorobots can effectively enter the body pits. After the section into the cells, nanorobots can be constrained by the specialist utilizing a PC. With the approach in the nanotechnology, 
numerous instruments are delivered on the nanoscale to do a medical procedure, even if it's just a single cell. Careful edges covered with nanoparticles, nanoneedles, nanolasers, nanocoated catheters, nanocontoured embed surfaces are the utilizations of nanotechnology in the medical procedure [7]. Nuclear Force Microscopy (AFM) can check the pictures in the nanoscale and discovers application in the imaging and therapeutics. AFM tips are around $20 \mathrm{~nm}$ or less and can create images with a high objective. These AFM pointers can do neighborhood medical procedure inside the cell level. The benefit of AFM advanced mechanics is that they can picture just as control the examples in nano scale. AFM-based nanorobots employ three approaches to overcome the limitations of traditional AFM. A joystick is used for movement control in the virtual reality interface. The joystick is equipped with threedimensional association powers that may be easily manipulated. To explore specific atomic cooperations, AFM tips can be functionalized with various ligands and antibodies. The use of explicit reagents coupled to AFM tips allows for in vitro local medication delivery. The visual input of living examples provides a continual web-based verification framework by detecting surface changes observed by the AFM tips [8].

\section{B. Nanorobots in Neurology}

In the realm of nanorobotics nervous system science, has been a functioning space of functional innovative work. The advancement of nanotechnology and the introduction of nanorobots can be beneficial in neurosurgery for a variety of reasons, including improved patient outcomes, location of pathology, insignificantly intrusive intracranial observing and drug conveyance and numerous others. The spinal rope and nerve injury is a genuine entanglement in the field of neurosurgery. Reconnection of cut across nerves and advancement of axon regeneration through improved platforms are the major challenges. The ability to repair can be triggered merely by reconnecting the damaged nerves. The physicians' restriction is to do the medical operation on that time scale. The use of nanoscale devices allows for the control of single axons. In an axon medical operation, a nano blade with a width of $40 \mathrm{~nm}$ was successful. Dielectrophoresis, an interaction that controls the polarizable objects in an electric field, constrains the mobility of axons in a careful field. Electrofusion or laser incited cell combination is then used to connect the two nerve endings [9]. The formation of a cluster in the blood arteries that provide oxygen to the cerebrum is known as cerebrum aneurysm. Because of the spilling or burst of the aneurysm, this severe draining intracranial illness is most commonly discovered between the foundation of the skull and under the cerebrum. Nanotechnology has contributed to the advancement of nano-sized robots for anticipating cerebrum aneurysms of cerewbrum The three key ideas of the development of nanorobots are hardware prototyping, fabricating approach and inside-body cooperation. The prototyping of clinical nanorobots requires the hardware prototyping which is set up with computational nanotechnology which drives as a critical device for the quick and successful improvement of instrumentation of robot. With the use of biomaterials, photonics, and nano bioelectronics, the assembly method focuses on creating a biochip coordinated robot. The nanorobots' resemblance to gel and contact with organic components and synthetic reactions is managed by the inside body association. The nanorobots' morphology is derived from cell morphology, proteomics, and microbiology. Nanorobots designed to detect aneurysms in the brain should be able to detect endothelial damage by tracking the veins before the subarachnoid discharge occurs. Because the small measured biomolecules are difficult to discriminate reliably, the compound nano biosensor aids the nanorobots in this cycle of placement.. Nanorobots designed to detect aneurysms in the brain should be able to detect endothelial damage by tracking the veins before the subarachnoid discharge occurs. The compound nano biosensor aids nanorobots in recognising the existence of intracranial aneurysm by measuring the amount of nitric oxide synthase (NOS) in the cerebrum. If the level of NOS exceeds a certain threshold,such as 100 proteomic signal transduction, it is a strong indicator of the presence of an intercranial aneurysm.Following that, the nanorobots provide data on the vessel's area as well as the bulb's element [10].

\section{Nanorobots in Dentistry}

Nano dentistry has become an arising field with the particular utilization of nanorobots at a critical everyday practice. The utilizations of nanorobots in the field of dentistry are cleaning, beautifying agents for teeth brightening, root trench treatment, treatment of extreme touchiness, arrangement of the teeth,improving the strength of the teeth, and oral absense of pain. The different tissue designing strategies are consolidated for the complex tooth fix. The oral absense of pain is set up by orally managing a combination including a huge number of nanorobots which can enter into gingival sulcus where it counts the mash. The nanorobots in a controlled way give the medication at the particular spaces of treatment. Root trench medicines and treatment of any dental contamination is made conceivable with the capacity of the nanorobots to be wrapped inside a case for cooperation and recognition. In the interaction of root channel treatment the utilization of minuscule camera inside the nanorobots for the perception of contaminated root supporting the specialist in the system makes the treatment a fruitful one. In 2011, the achievement pace of the root channel treatment is $70 \%$ as detailed National Health Service, which guarantees for development around here. Naturally, autologous entire tooth supplanting with nanostructured composite has totally upset dentition substitution treatment. Sapphire, a covalently fortified counterfeit material having multiple times the hardness strength than artistic what's more, standard brightening sealant has supplanted veneer in the counterfeit tooth. Nanocomposite is fabricated by nanoparticles dispersed in pitches. The nano filler is a blend of aluminum and silicon 


\section{International Journal of Engineering Applied Sciences and Technology, 2021 \\ Vol. 6, Issue 2, ISSN No. 2455-2143, Pages 236-239 \\ Published Online June 2021 in IJEAST (http://www.ijeast.com)}

powder with refractive file of 1.503 . It mixes well with normal tooth structure contrasted with the regular composites. The part of nanorobots in the treatment of dentine touchiness has expanded fascination. The quantity of dentinal tubules in touchy teeth is higher contrasted with the ordinary teeth. Nanorobots play out the particular removal when entered into the dentinal tubules which keeps the improvements from instigating the agony reaction. Nanorobots likewise help in dental care by the consolidation of nanorobots into the mouthwash,what's more, toothpaste which when utilized at a standard premise straightforwardly controls the periodontal tissues helping the cycle of tooth repositioning [9].

\section{Nanorobots in Hematology}

The uses of nanorobotics and nanomedicine in the field of hematology are at the exploration level and the applications are under investigation. The uses of nanorobots in hematology range from bondings of non-blood oxygen conveying to reestablishing essential hemostasis. Nanorobot named respirocyte is under plan for supporting the interaction of blood bonding. The size of the robot would be 1000 nanometers with its parts developed at nano level. It will contain an installed PC with a measurement of $58 \mathrm{~nm}$, oxygen and carbon dioxide stacking rotors with a breadth of $14 \mathrm{~nm}$. The three principle elements of nanorobot named respirocyte while going through the circulatory system are assortment of oxygen from the respiratory framework, assortment of carbon dioxide from tissues and utilizing the coursing glucose to control its own capacities. This robot is prepared to convey multiple times more oxygen per unit volume than the red platelets.

The Hemostasis is the second application where nanorbotics is engaged for research. The hemostasis includes a scope of steps which require different inhibitors adjusting the contaminated vein. The test looked in physiologic hemostasis is the normal draining time is almost 5 minutes. Nanorobotics can be utilized in tackling this issue. During the platelet bonding, the patients may foster contamination with microorganisms and prompting improvement of an insusceptible reaction. The fake mechanical platelet, "clottocyte" nanorobot has been proposed for mirroring this capacity. The size of the proposed robot is two micron alongside a lattice of $0.8 \mathrm{~nm}$, consolidated with hemostasis advancing protein which will be utilized for restricting the vessel injury during hemostasis.

The third possible use of nanorobots in the field of hematology is the part as phagocytic specialists. Nanorobots proposed are named as "Microbivores". Microbivore, an counterfeit mechanical phagocyte planned by Robert A. Freitas, is a nanorobotic gadget that securely destroys the blood-borne microbes. It is an oblate spheroidal nanomedical gadget containing 610 billion accurately masterminded primary particles in addition to 150 billion gas atoms. The nanorobot has various adaptable restricting locales on its outer surface for antigens and microorganisms. It is accepted to have the capability of relieving septicemia not long after organization and is more viable almost multiple times better compared to the regular phagocytic abilities. The acknowledgment of these nanorobotics applications can be useful in treatment of contaminations [10].

\section{E. Nanobots in Cancer Treatment}

With the headway of innovations in the clinical field, the therapy of disease can be effectively settled. The arly conclusion of the malignant growth has been the factor that chooses the odds of endurance of a disease patient. It is exceptionally essential to recognize the malignancy before metastasis stage for the fruitful therapy of malignancy. Nanorobots can be coordinated with compound biosensors for recognizing the presence of tumor at a beginning phase. E-Cadherin is a tumor silencer protein having a place with a class of transmembrane proteins. The Cadherin-1 otherwise called CAM $120 / 80$ is a phone grip atom and is reliant upon calcium particles to work. The power of ecadherin can be estimated by the nanorobots for recognizing the presence of tumor at the beginning phase. The nanorobots can be presented in tumor resection medical procedures. A radioactive colloid infusion will be controlled to the patient into the prostate daily before the resection medical procedure and radioisotope guided lymph hub analyzation will be performed which is all the more speedy in identifying the metastasis contrasted with open lymph analyzation. This method can be improved by the fuse of nanorobots wiping out the patient confirmation before the medical procedure [9].A significant part of disease treatment is the designated drug conveyance to decrease the symptoms of famous disease treatment, chemotherapy. As examined before, the nanorobots can go inside more modest vessels also, convey prescription at target locales. The nanorobots are profoundly potential to be utilized in malignancy treatment. The ordinary chemotherapy has the constraint in dose organization since it influences the ordinary cells alongside the tumor cells. The impediment can be overwhelmed by nanotechnology with the selectivity of treatment locales. Nanorobots can independently identify the malignancy cells and conveys the medication at the site of harmful regions. The nanorobot can be developed to specifically react to various cell surface receptors and the degree of payload to be regulated can likewise be controlled. The designed DNA strand is utilized for the development of this kind of nanorobot that has an ideal tertiary construction. The nanorobot after restricting with the wanted objective, unfurls its design to direct the portion at the destructive cells. Another nanorobot that has the potential to go along the vein and delivery the prescription at the objective region is worked of engineered components, called Pharmacyte. This nanorobot contains restorative payload having independent energy age and train capacity to get across the tissues and cell films [10]. 


\section{International Journal of Engineering Applied Sciences and Technology, 2021 \\ Vol. 6, Issue 2, ISSN No. 2455-2143, Pages 236-239 \\ Published Online June 2021 in IJEAST (http://www.ijeast.com)}

\section{CONCLUSION}

The nanorobots which alter the clinical field, helps in the determination and treatment of numerous infections counting malignancy. Nanorobots are site-explicit, nonobtrusive, PC control of conveyance and lessen the results brought about by the traditional treatment methods. This prompts the early analysis of sicknesses and improved treatment at low cost. The utilization of nanorobots for the ordinary analysis what's more, treatment is conceivable in not so distant future.

\section{ACKNOWLEDGMENT}

The authors would like to thank the management of Lingaya's Vidyaprrth, Faridabad, Haryana for their support and encouragement in publishing this paper

\section{REFERENCES}

[1] M. Apoorva, and V. Kishore, 2014"The Promising Future in Medicine: Nano robots", Sci. Educ., vol. 2(2), pp. 42-47.

[2] P. Khulbe, 2014, “Nanorobots: a review”, Int. J. Pharm. Sci. Res., vol. 5(6),p. 2164.

[3] Saxena.S,Pramod B.J, Dayananda B.C, and Nagaraju,K,2015 "Design,architecture and application of nanorobotics in oncology", Indian J.Cancer, vol. 52(2), pp. 236-241.

[4] Cavalcanti.A, Shirinzadeh.B,Zhang.M, and Kretly.L.C,2008."Nanorobot hardware architecture for medical defense", Sensors, vol. 8(5), pp.2932-2958.

[5] S.M.M.R. Al-Arif, Quader.N, Shaon.A.M , and Islam.K.K,2011, "Sensor based autonomous medical nanorobots: A cure to demyelination", J.Sel. Areas Nanotechnol., pp.10-15.

[6] Cavalcanti.A, Shirinzadeh.B, Freitas Jr.R.A. and Hogg.T,2007, "Nanorobot architecture for medical target identification", Nanotechnology, vol. 19(1), p.015103.

[7] Mali.S,2013 "Nanotechnology for surgeons", Indian J. Surg., vol. 75(6), pp.485-492.

[8] Song.B, Yang.R, Xi.N,Patterson K.C, Qu.C, and Lai. K.W.C,2012,“Cellular-level surgery using nano robots”, J. Lab. Autom., vol. 17(6),pp. 425-434.

[9] Saadeh.Y, and Vyas.D,2014 "Nanorobotic applications in medicine:current proposals and designs", Am. J. Robotic Surg., vol. 1(1), pp. 4-11.

[10] Gupta.J ,2011"Nanotechnology applications in medicine and dentistry", vol. 2(2), pp. 81-88.

[11] Bharghava A, Cheung J, EshaghianWilner MM, et al.Review of nanomedicine. Internation Journal Nano Studies Technology. 2016;5(10):100-101.

[12] Malasala Satyaveni. et al. / International Journal of Biological \& Pharmaceutical Research. 2013; 4(4): 297301.

[13] Li.M.,Xi.N, WMang.Y, and Liu.L,2020 "Progress in nanorobotics foradvancing biomedicine", IEEE Trans. Biomed. Eng., pp. 1-18, 